\title{
CALCULATION AND SIMULATION OF AERODYNAMIC CHARACTERISTICS OF WIND POWER PLANT TURBINE
}

\author{
Aleksandrs Urbahs, Nikolaj Kuleshov, Dmitrijs Titovs, Leonid Chernin \\ Riga Technical University, Latvia \\ aleksandrs.urbahs@rtu.lv, nikolajs.kulesovs@rtu.lv,dmitrijs.titovs@rtu.lv, 1.chernin@gmai.com
}

\begin{abstract}
The physics of a perturbed flow around the wind power plant turbine is rather complex. When passing through the plane of rotation, the airflow gets substantially perturbed. In such conditions, the pressure of ram air changes stepwise when passing through the plane of the wind turbine rotation, which is related to the loss of flow mechanical energy and its transfer to the rotor. A complex vortex system is created behind the turbine. In this system, it is possible to single out at least three distinctive groups of vortices: attached vortices on blades, a free helicoidal vortex that forms when the flow is passing through the blade ends, and an axial vortex forming at the expense of flow swirling on the wind turbine. In a real flow, the whole perturbed area is filled with vortices, which makes it quite difficult to perform a physical analysis and mathematical calculation of the flow. The article presents the results of calculating and simulating the aerodynamic characteristics of a propeller-type wind turbine with a horizontal axis of rotation. The calculations are based on the theory of elementary streams, which is a compilation of the results of the momentum theory of the ideal wind turbine and a wing theory, which is quite well developed now. On this basis, it is possible to substantially expand the study and description of physical processes related to wind turbine flow. The rated power of the wind power plant, rated wind speed, wind speed at gusts, number of blades, etc. were taken as initial data. For the selected wind turbine blade airfoil of "Espero" type an angle of attack, a lift coefficient and a drag-to-lift ratio were determined. During the calculation and simulation the cases of an airfoil with and without a geometric twist were considered. As a result of the calculation and computer simulation, there were determined the optimal values of the coefficients of: flow deceleration, ideal power, end and profile losses, blade tip specific speed.
\end{abstract}

Keywords: wind, power, plant, turbine, blades, aerodynamics, geometry.

\section{Introduction}

The physics of a perturbed flow around the wind turbine is rather complex [1-5]. When passing through the plane of rotation, the airflow gets substantially perturbed. At the same time, there appears a rotational velocity $U$, which is equal to zero in ram air, while the longitudinal velocity $V$ decreases. It is also possible to point out other wind turbine operating modes, for example, the propeller mode. In this case, on the contrary, the longitudinal velocity in the perturbed flow behind the wind turbine will be higher due to the supply of mechanical energy to the flow. The aerodynamic calculation of wind turbine is related to only those modes at which the wind turbine receives energy from ram air. In such conditions the pressure changes stepwise, when passing through the plane of the wind turbine rotation, which is related to the loss of flow mechanical energy and its transfer to the turbine. At a long distance up and down the flow the pressure coincides with the atmospheric pressure.

A complex vortex system is created behind the turbine. In this system it is possible to single out at least three distinctive groups of vortices: bound vortices on blades, a free helicoidal vortex that forms when the flow is passing through the blade ends, and an axial vortex forming at the expense of flow swirling on the wind turbine. In a real flow, the whole perturbed area is filled with vortices, which makes it quite difficult to perform a physical analysis and mathematical calculation of the flow.

The first theoretical developments in the field of wind-powered engine studies were based on the so-called momentum theory, as the determination of forces and moments acting upon the rotor was based on the laws of mass conservation, motion quantity and energy. It is clear that such a theory could not provide information about the geometrical parameters of the wind turbine, its structure and operating modes; however, this theory helped evaluate the efficiency factor of the wind turbine and the flow rate.

Later on, a theory of elementary streams was created, which is a compilation of the results of the momentum theory of the ideal wind turbine, and the wing theory, which is quite well developed now. On this basis, it is possible to substantially expand the study and description of physical processes related to the wind turbine flow. 
The article presents the results of calculating and simulating the aerodynamic characteristics of a propeller-type wind turbine with a horizontal axis of rotation. The calculations are based on the theory of elementary streams.

\section{Materials and methods}

Applying the theory of elementary streams for calculating the propeller-type wind turbine, it is necessary to take into consideration the non-uniform distribution of velocities at which the air flows through the wind turbine, the relation of these velocities to the geometrical parameters of the blades and their aerodynamic characteristics.

For this purpose, on the one hand, it is required to determine the forces acting upon the wind turbine by studying wake flotation on the basis of the momentum theory of the ideal wind-powered engine; on the other hand, it is necessary to determine these forces from the ratios for each blade airfoil.

When the air flow acts upon the wind turbine with a velocity $v$, aerodynamic forces rotating the wind turbine occur on the blades (Figs. 1 and 2). The flow runs over each blade element with a relative velocity $W$ at an angle $\alpha$, which is called an angle of incidence (Fig. 2).

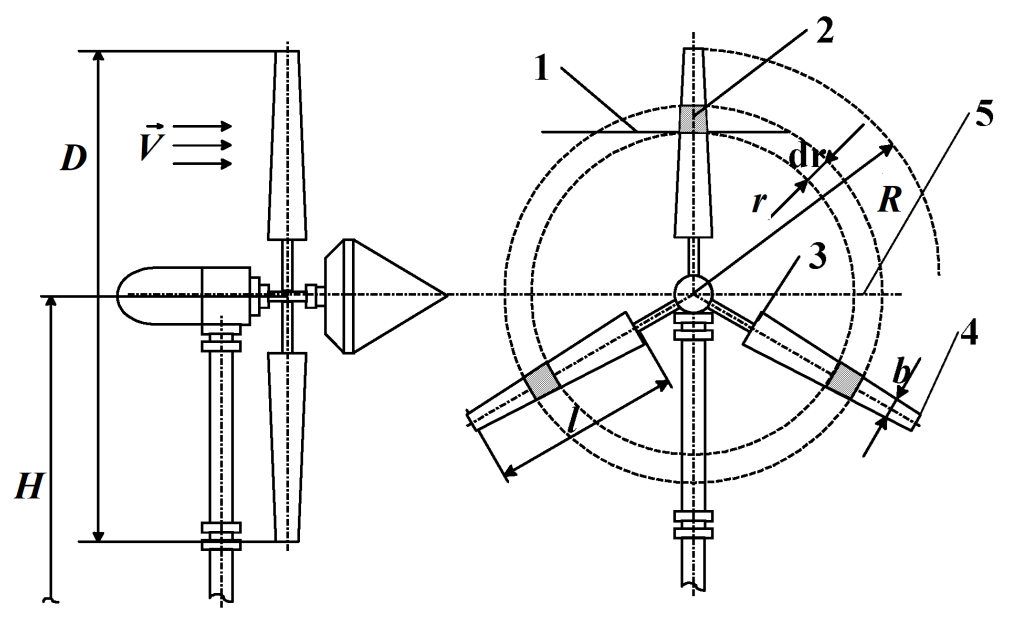

Fig. 1. Diagram of propeller-type wind turbine: 1 - intermediate section; 2 - elementary blade;

3 - root section; 4 - peripheral section; 5 - elementary annular stream

Expressions for these forces contain unknown induced velocities $u_{1}$ and $v_{1}$, occurring as a result of the effect of the rotating blades on the flow (Fig. 2). Equalization of ratios for the forces obtained by both methods leads to a closed system of equations relative to induced velocities.

Knowing the induced velocities $u_{1}$ and $v_{1}$ in the flow, it is possible to calculate the axial force and driving torque coefficients.

- The axial force coefficient:

$$
C_{y_{a}}=8 \int_{\overline{\bar{r}}_{k}}^{1}\left(1-\bar{v}_{1}\right) \overline{v_{1}} \bar{r} d \bar{r}
$$

where $\quad \bar{v}_{1}=\frac{v_{1}}{v}-$ the relative induced velocity of the wind;

$v_{1}$ - induced velocity of the wind;

$r$ - radius of the wind turbine element being considered (see Fig. 1);

$d r$ - length of the wind turbine element being considered (see Fig. 1);

$r_{k}$ - radius of the root section of the wind turbine blade.

- The driving torque coefficient:

$$
C_{M}=8 \int_{\bar{v}_{k}}^{1}\left(1-\bar{v}_{1}\right) \bar{u}_{1} \bar{r}^{2} d \bar{r}
$$


where $\quad \bar{u}_{1}=\frac{u_{1}}{u}-$ the relative induced rotational velocity;

$u_{1}$ - induced rotational velocity.

The wind turbine power coefficient

$$
C_{P}=8_{z R} \int_{\bar{v}_{k}}^{1}\left(1-\bar{v}_{1}\right) \bar{u}_{1} \bar{r}^{2} d \bar{r},
$$

where $z_{R}$-wind turbine speed coefficient.

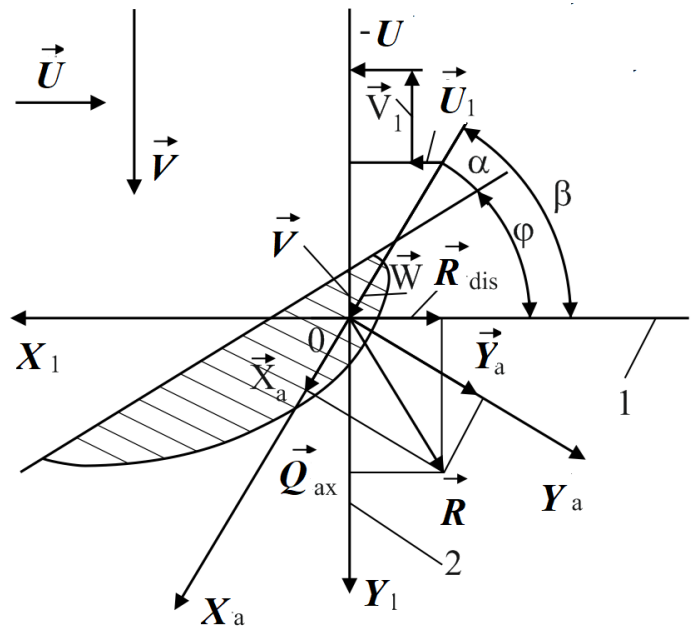

Fig. 2. Blade airfoil, speed plan and reaction forces: 1 - plane of rotation; 2 - wind turbine axis

Knowledge of the listed coefficients provides an opportunity to design wind-powered engines with the required parameters.

Thus, the theory of elementary streams takes into consideration the non-uniform distribution of velocities at which the air flows through the wind turbine, the relation of these velocities to the geometrical parameters of the blades and their aerodynamic characteristics (1-3).

The proposed calculation method is based on two constraint equations stemming from the method of elementary streams schematically described above [6-9]. The first constraint equation stems from the fact that the axial component of the force of flow reaction to elementary blades located in the action area of elementary annular stream (Fig. 1) is equal to the force resulting from the effect of pressure difference in front of and behind the turbine upon the area of elementary annular stream section by the turbine rotation plane. This constraint leads to the following equation:

$$
i_{b l} \bar{b} C_{y_{a}}=\frac{8 \pi \bar{r} e}{(1+e)(1-e)^{2}\left(z_{u}+\mu_{a}\right) \sqrt{1+z_{u}^{2}}},
$$

where $e$-coefficient of flow deceleration;

$i_{b l}$ - number of wind turbine blades;

$\bar{b}$ - chord of blade airfoil in the pre-set section;

$z_{u}$ - number of the relative moduli of the blade section;

$\mu_{a}$ - drag-to-lift ratio.

Using the selected geometrical parameters of the blade and its pre-set angles, the ratio (4) allows to determine the coefficient of flow deceleration $e$ on the given radius $r$ depending on the angle of incidence $\alpha$.

The second constraint equation reflects the principle of angular momentum, which, as applied to the wind turbine, can be formulated in the following way: the wind turbine axis-related momentum of the aerodynamic forces acting upon the elementary blades (Figs. 1,2) is equal in value and opposite in sign to the moment of momentum received by the elementary annular stream entrained by the wind turbine. The second constraint equation leads to the following: 


$$
z=z_{u}(1-e)-\frac{e}{1+e} \cdot \frac{1-\mu_{a} z_{u}}{z_{u}+\mu_{a}},
$$

Equation (5) makes it possible to determine the number of moduli $z$ from the determined values $e$ and $\beta=\varphi+\alpha$, where $\varphi$ is the blade angle (determined by the wind turbine structure) (see Fig. 2). Constraint equations (4) and (5) form a basis for the aerodynamic calculation of the wind turbine.

\section{Results and Discussion}

Initial data for calculating the wind turbine with a horizontal axis of rotation are presented in Table 1. Besides, it is also required to determine the type of the turbine blade section airfoil, its geometrical and aerodynamic characteristic. An airfoil of "Espero" type with 15 percent thickness was selected for the calculation (Fig. 3) [7]. The aerodynamic and geometric parameters of the selected airfoil were determined for 15 points uniformly distributed along the chord $\mathrm{b}$ of the section (see Fig. 3). The first point coincides with the origin of coordinates (see Fig. 3). The values obtained selectively for five points are shown in Tables 2 and 3.

Initial data for wind turbine calculation

Table 1

\begin{tabular}{|c|c|c|}
\hline Parameter name & Designation & Value \\
\hline Rated power & $P$ & $3000 \mathrm{~W}$ \\
\hline Design wind speed & $V$ & $7.5 \mathrm{~m} \cdot 10 \mathrm{~s}^{-1}$ \\
\hline Gust speed & $V_{\text {gust }}$ & $25 \mathrm{~m} \cdot 10 \mathrm{~s}^{-1}$ \\
\hline Number of wind turbine blades & $i_{b l}$ & 3 \\
\hline Air density under normal atmospheric conditions & $\rho$ & $1.24 \mathrm{~kg} \cdot 10 \mathrm{~m}^{-}$ \\
\hline $\begin{array}{c}\text { Pre-set speed coefficient at the tip of the blade at the } \\
\text { wind turbine driving-point }\end{array}$ & $z_{R}$ & 6.5 \\
\hline Relative radius of blade root section position & $\bar{r}_{0}$ & 0.3 \\
\hline $\begin{array}{c}\text { Design number of blade sections } \\
\text { fumber of division points in the specified interval }\end{array}$ & $n$ & 21 \\
\hline $\begin{array}{c}\text { coefficing the optimum value of the deceleration } \\
\text { coefient }\end{array}$ & $n_{e}$ & 20 \\
\hline
\end{tabular}

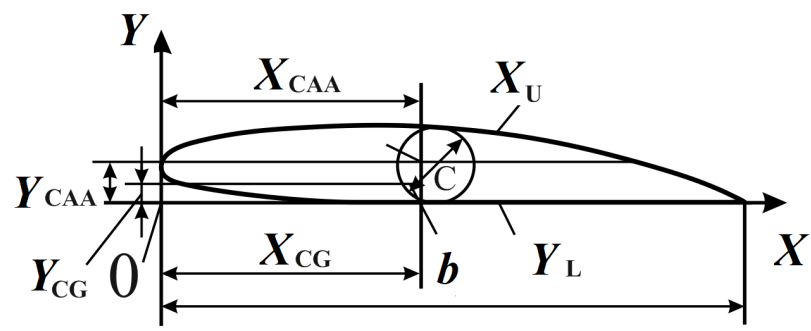

Fig. 3. Blade airfoil geometry

Aerodynamic characteristics of "Espero" airfoil

\begin{tabular}{|c|c|c|c|}
\hline $\begin{array}{c}\text { Point number } \\
\text { on the airfoil chord }\end{array}$ & $\begin{array}{c}\text { Angle of incidence } \\
\boldsymbol{\alpha}\end{array}$ & $\begin{array}{c}\text { Lift coefficient } \\
\boldsymbol{C} \boldsymbol{y} \boldsymbol{\alpha}\end{array}$ & $\begin{array}{c}\text { Drag-to-lift ratio } \\
\boldsymbol{\mu} \boldsymbol{\alpha}\end{array}$ \\
\hline 1 & $-6^{\mathbf{o}}$ & 0.10 & 0.1250 \\
\hline 5 & $1^{\mathbf{o}}$ & 0.76 & 0.0197 \\
\hline 9 & $8^{\mathbf{o}}$ & 1.16 & 0.0410 \\
\hline 12 & $14^{\mathbf{o}}$ & 1.13 & 0.1217 \\
\hline 15 & $20^{\mathbf{o}}$ & 0.99 & 0.2626 \\
\hline
\end{tabular}


Dimensionless coordinates of "Espero" airfoil

Table 3

\begin{tabular}{|c|c|c|c|}
\hline $\begin{array}{c}\text { Point number } \\
\text { on the airfoil chord }\end{array}$ & $\overline{\boldsymbol{x}}=(\boldsymbol{x} / \boldsymbol{b}) \cdot \mathbf{1 0 0} \%$ & $\begin{array}{c}\text { Upper limit } \\
\overline{\boldsymbol{y}}_{\boldsymbol{H}}=\left(\boldsymbol{y}_{\boldsymbol{H}} / \boldsymbol{c}\right) \cdot \mathbf{1 0 0} \boldsymbol{\%}\end{array}$ & $\begin{array}{c}\text { Lower limit } \\
\overline{\boldsymbol{y}}_{\boldsymbol{L}}=\left(\boldsymbol{y}_{\boldsymbol{L}} / \boldsymbol{c}\right) \cdot \mathbf{1 0 0} \boldsymbol{\%}\end{array}$ \\
\hline 1 & 0 & 41.7 & 41.7 \\
\hline 5 & 10 & 82.2 & 11.1 \\
\hline 9 & 50 & 94.5 & 0 \\
\hline 12 & 80 & 51.0 & 0 \\
\hline 15 & 100 & 2.3 & 0 \\
\hline
\end{tabular}

The following parameters were also determined for the selected "Espero" airfoil:

- centre of gravity $(C G)$ coordinates and related characteristics $\left\{\begin{array}{l}x_{C G}=0.352 b ; \\ y_{C G}=0.4 c ;\end{array} \Leftrightarrow\left\{\begin{array}{l}x_{C G} / b \cdot 100 \%=35.2 \% ; \\ y_{C G} / c \cdot 100 \%=40 \% .\end{array}\right.\right.$

- coordinates of the centre of airfoil alignment $(C A A)$ and related characteristics $\left\{\begin{array}{l}x_{C A A}=0.352 b ; \\ y_{C A A}=0.5 c ;\end{array} \Leftrightarrow\left\{\begin{array}{l}x_{C A A} / b \cdot 100 \%=35.2 \% ; \\ y_{C A A} \Pi / c \cdot 100 \%=50 \% .\end{array}\right.\right.$

- $\quad$ value of the parameter $c$ (see Fig. 3) is 0.156 .

To determine the optimum value of the flow deceleration coefficient, it is required to pre-calculate a number of parameters (Table 4 ).

Table 4

Calculation formulas for determining wind turbine parameters

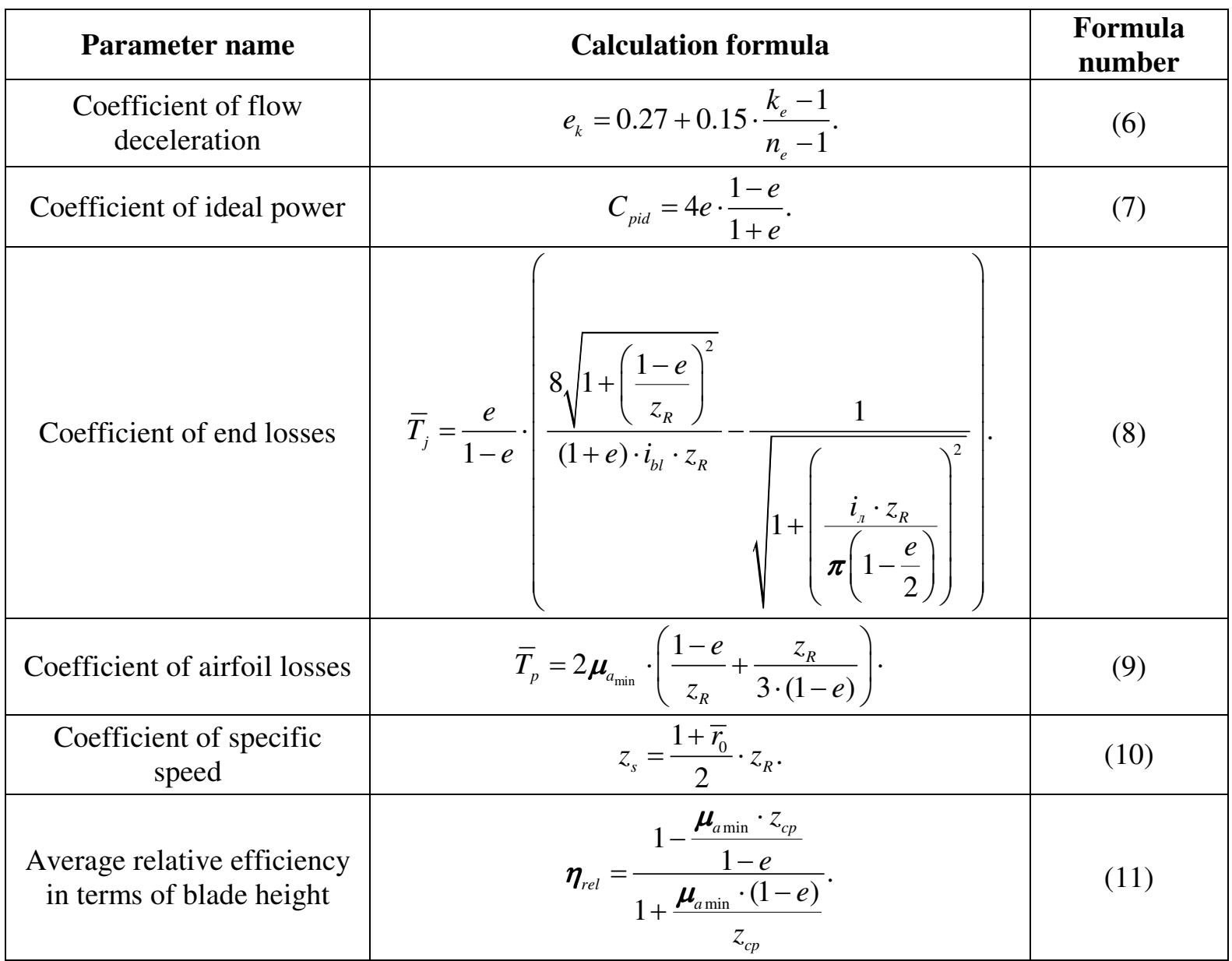


Table 4 (continued)

\begin{tabular}{|c|c|c|}
\hline Parameter name & Calculation formula & $\begin{array}{c}\text { Formula } \\
\text { number }\end{array}$ \\
\hline $\begin{array}{c}\text { Coefficient of stream twist } \\
\text { losses }\end{array}$ & $\bar{T}_{m}=C_{p i d} \boldsymbol{\eta}_{r e l}^{2} \cdot \frac{\ln \left(1 / \bar{r}_{0}\right)}{2 z_{R}^{2}}$. & (12) \\
\hline Preliminary power coefficient & $C_{p_{p p}}=C_{p_{i d}} \cdot\left(\left(1-\bar{d}_{0}^{2}\right)-\bar{T}_{j}-\bar{T}_{p}-\bar{T}_{m}\right)$. & (13) \\
\hline
\end{tabular}

According to formula (6) (see Table 4), using the known $n_{e}=20$ (see Table 1) and the prescribed $k_{e}=1 \ldots 20$, it is possible to obtain a number of values of the flow deceleration coefficient in the interval from 0.270 to 0.420 . Further calculations according to formulas (7) ...(13) were performed for twenty obtained values of $e_{k}$. The mean value of the coefficient of specific speed was 4.225 (formula (10), Table 4). The optimum value of the coefficient of flow deceleration $e_{o p t}$ corresponds to the maximum value of the preliminary power coefficient $C_{p p}$ from the obtained design set for the average (in terms of radius) coefficient of the specific speed $\mathrm{z}_{\mathrm{s}}$. According to the calculation results, the maximum value of the preliminary power coefficient $C_{p p}$ was 0.462 , and the corresponding optimum value of the coefficient of flow deceleration $e_{\text {opt }}=0.333$. The coefficient of ideal power calculated by formula (7) (see Table 4) for the case $e_{\text {opt }}$ was 0.666.

The calculation of the blade geometry parameters (Table 5) was performed for 8 sections, starting with the root $(r=0.3)$ and finishing with the peripheral $(r=1.0)$ section.

Table 5

Calculation formulas for determining blade geometry parameters

\begin{tabular}{|c|c|c|}
\hline Parameter name & Calculation formula & $\begin{array}{c}\text { Formula } \\
\text { number }\end{array}$ \\
\hline $\begin{array}{c}\text { Coefficient of blade section } \\
\text { specific speed }\end{array}$ & $z_{k}=z_{R} \cdot \bar{r}_{k} \cdot$ & $(14)$ \\
\hline $\begin{array}{c}\text { Number of blade section } \\
\text { relative moduli }\end{array}$ & $Z_{U k}=Z_{k} \cdot \frac{1+\sqrt{1+\frac{C_{p i d}}{Z_{k}^{2}}}}{2\left(1-e_{o p t}\right)}$. & $(15)$ \\
\hline $\begin{array}{c}\text { Total loading coefficient of the } \\
\text { sections of those blades which } \\
\text { are located in the action area of } \\
\text { elementary annular stream }\end{array}$ & $C_{l_{k}}=\frac{1}{\left(1+e_{o p t}\right)\left(1-e_{o p t}\right)} \cdot \frac{1}{\left(z_{u_{k}}+\mu_{a_{\min }}\right) \sqrt{1+z_{u_{k}}^{2}}}$ & $(16)$ \\
\hline $\begin{array}{c}\text { Lift coefficient of peripheral } \\
\text { section }\end{array}$ & $C_{y a p}=C_{y_{a}}\left(\mu_{a_{\min }}\right)$. & \\
\hline $\begin{array}{c}\text { Relative chord of peripheral } \\
\text { section (in fractions of turbine } \\
\text { outer radius) }\end{array}$ & $\bar{b}_{p}=\frac{C_{l_{p}}}{i_{b i} \cdot C_{y_{a} p}}$. & $(17)$ \\
\hline Inflow angle of blade section & $\boldsymbol{\beta}_{k}=\operatorname{arctg}\left(1 / z_{u_{k}}\right)$. & $(19)$ \\
\hline Pitch angle of blade section & $\boldsymbol{\varphi}_{k}=\boldsymbol{\beta}_{k} \boldsymbol{\alpha}_{k}$ & $(20)$ \\
\hline
\end{tabular}

In expressions (16) and (17), $\mu_{\text {amin }}$ is the minimum value of the drag-to-lift ratio (it is taken from the aerodynamic characteristics of the selected airfoil and is 0.018).

For the selected airfoil, it is possible to obtain the value of the peripheral section lift coefficient $C_{\text {yap }}=0.85$.

In case of using a tapered blade, the distribution of $C_{y a}$ differs from the optimum value 0.85 . As modern technology makes it possible to produce blades of any shape, it is expedient to specify the maximum value $C_{y \max }=0.85$, which is equal for all sections, rather than the chord. Accordingly, the angle of incidence for all sections is constant $\alpha=2^{\circ}$, and the value of section chords determined based on expression (18) (see Table 5) is: $0.0574 \mathrm{~m}$ for the peripheral section, $0.169 \mathrm{~m}$ for the root section. 
Relative airfoil thicknesses $c_{\text {rel }}$ for the root and peripheral sections were taken as equal to 0.1 and 0.25 respectively. The intermediate relative thicknesses are calculated by the law of chord length variation.

The inflow angle of the blade section can be determined by formula (19) (see Table 5). For the root and peripheral sections we can respectively obtain $\beta_{k r}=18.17$ and $\beta_{k p}=5.84$.

The pitch angle of the blade section determined by formula (20) (see Table 5) for the root and peripheral sections is $\varphi_{k r}=16.17$ and $\varphi_{k p}=3.84$ respectively.

According to the results of calculations, the wind turbine blades with a geometric twist lengthwise of the blade (Fig. 4a) and without a twist (Fig. 4.b) were designed in SolidWorks. Figure 5 shows the end view of the blade airfoil sections for the relevant blades.

a)

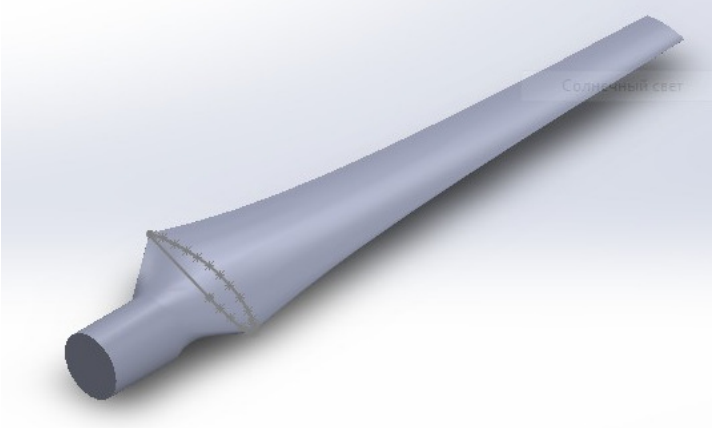

b)

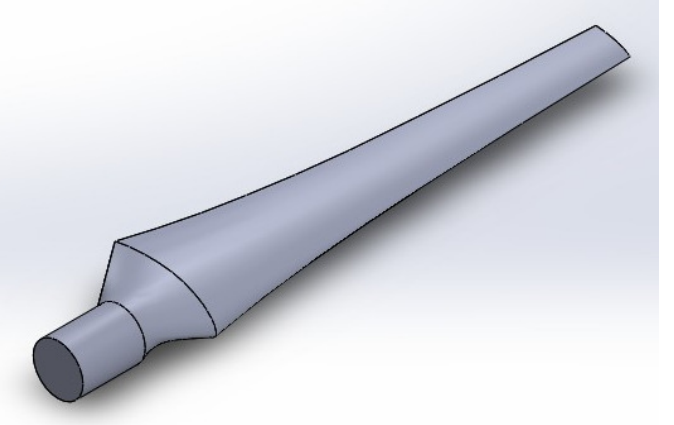

Fig. 4. Wind turbine blades with geometric twist (a) and without twist (b)

a)

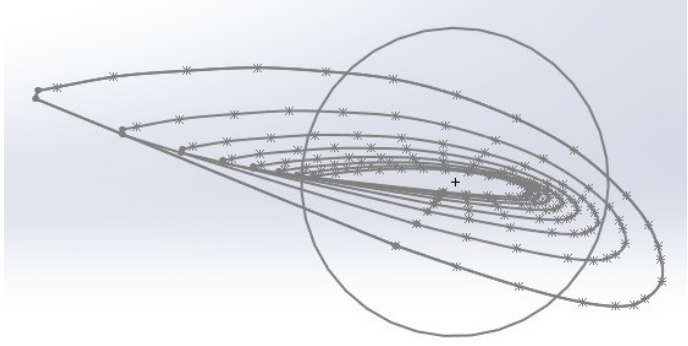

b)

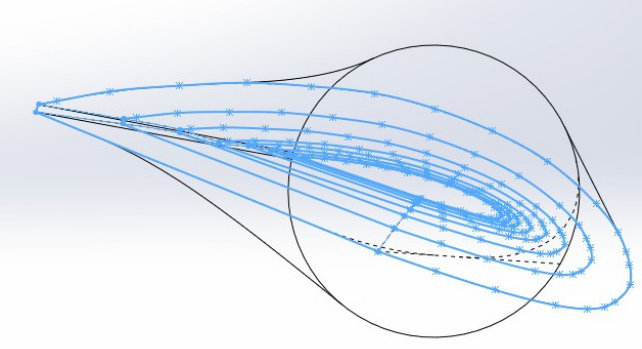

Fig. 5. End view of blade airfoil sections: $a$ - with geometric twist; $b$ - without geometric twist

To consider the effect of the wind turbine power and the wind speed, it is expedient to test a geometrically similar model in a wind tunnel. As a result, it will become possible to determine the dependencies of the torque coefficient and wind yield coefficient on the coefficient of specific speed. Then, based on similarity, the required wind turbine radius can be determined. Blade geometry, in this case, remains the same.

\section{Conclusions}

1. The article presents the results of calculating and simulating the aerodynamic characteristics of a propeller-type wind turbine with a horizontal axis of rotation. The calculations are based on the theory of elementary streams, which is a compilation of the results of the momentum theory of the ideal wind turbine and the wing theory, which is quite well developed now.

2. For the selected turbine blade airfoil of "Espero" type the angle of incidence, the lift coefficient and the drag-to-lift ratio have been determined. During the calculation and simulation, the cases of an airfoil with and without a geometric twist were considered.

3. The rated power of the wind power plant, design wind speed, gust speed, number of blades, etc., have been taken into consideration as initial data.

4. As a result of the calculation and computer simulation, there have been determined the optimal values of the coefficients of: flow deceleration, ideal power, end and profile losses, blade tip specific speed. 


\section{Acknowledgements}

This work has been supported by the European EUROSTARS project E9349 - OptiSWT "A direct drive SWT with aerodynamic pitch control of wind rotor blades and yaw oscillation damper (OptiSWT)".
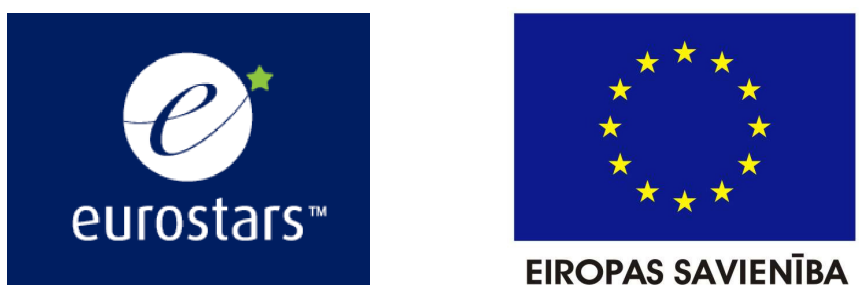

EIROPAS SAVIENĪBA

\section{References}

[1] Bilgili M., Yasar A., Ilhan A., Sahin B. Aerodynamic Characteristics of a Horizontal Axis Wind Turbine in Belen-Hatay, Turkey. International journal of Natural and Engineeing Science, vol. 9(1), 2015, pp. 54-58.

[2] Lee S, Kim H, Lee S. Analysis of aerodynamic characteristics on a counter-rotating wind turbine, Current Applied Physics. 2010, pp.339-342.

[3] Kishinami K, Taniguchi H, Suzuki J, Ibano H, Kazunou T, Turuhami M. 2005. Theoretical and experimental study on the aerodynamic characteristics of a horizontal axis wind turbine, Energy 2005, pp. 2089-2100.

[4] Burton T, Jenkins N, Sharpe D, Bossanyi E. 2011. Wind Energy Handbook", John Wiley \& Sons Ltd., United Kingdom, 2011.

[5] Urbahs, A., Urbaha M., Carjova K. Development and assessment of planetary gear unit for experimental prototype of vertical axis wind turbine. Latvian Journal of Physics and Technical sciences, Vol.5, 2017, pp. 48-56.

[6] Фатеев Е.M. Ветродвигатели и ветроустановки (Wind Engines and Wind Turbines), 1957, 544 p. (In Russian).

[7] Сабинин Г.X. Теория регулирования быстроходных ветродвигателей поворотом лопастей центробежным регулятором (A Theory of Controlling High Speed Wind Engines by Turning Blades with a Centrifugal Governor), Труды ЦАГИ, 1957, № 8, pp. 5-77. (In Russian)

[8] Hansen, Martin O. L. Aerodynamics of Wind Turbines, 2nd edition. Abingdon, Oxon: Routledge; 2013

[9] Erich Hau. Wind Turbines: Fundamentals, Technologies, Application, Economics. Springer Science \& Business Media, 2005, 783 p. 